\title{
ON BOUNDED UNIVALENT FUNCTIONS WHOSE RANGES CONTAIN A FIXED DISK
}

\author{
BY
}

ROGER W. BARNARD

\begin{abstract}
Let $\delta$ denote the standard normalized class of regular, univalent functions in $K=K_{1}=\{z:|z|<1\}$. Let $\mathscr{F}$ be a given compact subclass of $\delta$. We consider the following two problems. Problem 1. Find $\max \left|a_{2}\right|$ for $f \in \mathscr{F}$. Problem 2. For $|z|=r<1$, find the $\max (\min )|f(z)|$ for $f \in \mathscr{F}$. In this paper we are concerned with the subclass $\delta_{d}^{*}(M)=\left\{f \in \mathcal{S}: K_{d} \subset\right.$ $f(K) \subset K_{M}$. Through the use of the Julia variational formula and the Loewner theory we determine the extremal functions for Problems 1 and 2 for the class $\delta_{d}^{*}(M)$, for all $d, M$ such that $\frac{1}{4}<d<1<M<\infty$.
\end{abstract}

I. Introduction. Let $\delta$ denote the class of functions $f(z)=z+a_{2} z^{2}+\ldots$, that are regular and univalent in $K=K_{1}=\{z:|z|<1\}$. Let $\mathscr{F}$ be a given compact subclass of $\delta$. We consider the following two problems.

Problem 1. Find $\max \left|a_{2}\right|$ for $f(z) \in \mathscr{F}$.

Problem 2. For $|z|=r<1$, find $\max (\min )|f(z)|$ for $f(z) \in \mathscr{F}$.

It is well known [4] that for $f(z)$ in $\delta, f(K)$ contains $K_{1 / 4}$ and the function $k(z)=z /(1-z)^{2}$ solves Problems 1 and 2 for the class $\delta$ and hence for the subclass $\delta^{*}$ of starlike functions in $\delta$.

In [14] T. Suffridge solved Problem 1 for the class $\delta_{d}^{*}$ where $\delta_{d}^{*}=\{f \in$ $\left.\delta^{*}: f(K) \supset K_{d}\right\}$. He showed that an extremal domain for Problem 1 for $\delta_{d}^{*}$, $\frac{1}{4}<d<1$, has as its boundary a single arc $A C$ of $\partial K_{d}$ and two radial rays from $A$ and $C$ to $\infty$. In this paper Suffridge also conjectured that an extremal domain for Problem 1 in the class $\delta_{d}=\left\{f \in \delta: f(K) \supset K_{d}\right\}$ is the domain having as its boundary a single arc $A C$ of $\partial K_{d}$ and a radial slit to $\infty$ from the midpoint of $A C$. E. Netanyahu [11] verified this conjecture.

In this paper we will be concerned with the subclass $\delta_{d}^{*}(M)$ of $\delta$, $\delta_{d}^{*}(M)=\left\{f \in \delta^{*}: K_{d} \subset f(K) \subset K_{M}\right\}$. If we let $\gamma(M)$ be the radius of the Koebe disk for $\mathcal{S}^{*}(M)$, i.e., the largest disk with center $w=0$, contained in $f(K)$ for all $f \in \mathcal{S}^{*}(M)$, we need only consider $d \geqslant \gamma(M)$ for the class $\delta_{d}^{*}(M)$. As is well known, $\gamma(M) \rightarrow \frac{1}{4}$ as $M \rightarrow \infty$, while $\gamma(M) \rightarrow 1$ as $M \rightarrow 1$. Schwarz's lemma shows that for $f \in \mathcal{S}_{d}^{*}(M), f(K)$ cannot contain $K_{d}$ with $d>1$ and the only function in $\delta_{1}^{*}(M)$, or $\delta_{d}^{*}(1)$ is $f(z)=z$.

Received by the editors March 29, 1973 and, in revised form, August 6, 1975.

AMS (MOS) subject classifications (1970). Primary 30A72.

Key words and phrases. Univalent, starlike, distortion theorems, variational techniques. 
In this paper we shall, through the use of variational methods, determine the extremal functions for Problems 1 and 2 for the class $\delta_{d}^{*}(M)$.

We observe that Suffridge's result follows from ours when $M=\infty$. It is immediate that if the extremal function $f^{*}(z)$ solving Problem 2 is the same for all $r$ sufficiently close to zero, then $f^{*}(z)$ also solves Problem 1 . As we shall see the extremal function for Problem 2 in $\mathcal{S}_{d}^{*}(M)$ has this property and thus our results generalize Suffridge's result.

In $\S \S I I$ and III of this paper we introduce the Julia variational formula and show how it can be utilized to solve Problem 2 for the classes of functions under consideration. In these two sections, we will frequently need to use the concept and properties of the conformal mapping radius of a domain. The conformal mapping radius of a simply connected domain $\Omega$ with respect to $w=0$ (denoted m.r. $\Omega$ ) is defined as follows: If $w=f(z)=R z+a_{2} z^{2}$ $+\ldots$ maps $K, 1-1$, onto $\Omega=f(K)$, with $R>0$, then $R=$ m.r. $\Omega$.

In $\S I V$ we introduce additional variational methods that will be needed to study Problem 2 for the class $\delta_{d}^{*}(M)$. In particular, we show how to combine the Julia variational formula with the Loewner slit mappings to produce variations within the class $\delta_{d}^{*}(M)$.

In $\S \mathrm{V}$, we combine the variational methods of $\S I \mathrm{~V}$ with those of $\S I I I$ to solve Problem 2 for $\delta_{d}^{*}(M)$. We prove that the boundary of an extremal domain giving the solution to Problem 2 in $\delta_{d}^{*}(M), M<\infty$, consists of an arc $A C$ of $\partial K_{d}$, two radial sides $A B$ and $C D$, and the arc $B D$ of $\partial K_{M}$ connecting $B$ to $D$.

A routine mapping radius argument as in [1] shows that the extremal domain described for $\mathcal{\delta}_{d}^{*}(M)$ is in fact the range of a function in $\delta_{d}^{*}(M)$. We let $f^{*}(z, d, M)$ denote this function. We can determine $f^{*}(z, d, M)$ implicitly as follows. Consider the function

$$
\begin{aligned}
F(z)= & z f^{* \prime}(z) / f^{*}(z) \\
= & \left\{\left[a\left(z+z^{-1}\right)+b+2\right] /\left[a\left(z+z^{-1}\right)+b-2\right]\right\}^{1 / 2}, \\
& F(0)=1,
\end{aligned}
$$

where $a \geqslant b / 2+1, b \geqslant 0 . F(z)$ is found by a sequence of elementary transformations mapping $K$ onto the right half-plane $\operatorname{Re}\{w\}>0$ minus the slits $\{w: 0<w<c\}$ and $\{w: w \geqslant d\}$ where

$$
d=[(b+2 a+2) /(b+2 a-2)]^{1 / 2}
$$

and

$$
c=[(b-2 a+2) /(b-2 a-2)]^{1 / 2} \text {. }
$$

Since $\operatorname{Re}\{w\}>0, f^{*}(z)$ is in $\mathcal{S}^{*}$.

Let $\phi_{1}$ and $\phi_{2}$ be defined such that $0<\phi_{1}<\phi_{2}<\pi$ and $\lim _{r \rightarrow 1} f\left(r e^{i \phi_{1}}\right)=$ 
$\infty$ and $F\left(e^{i \phi_{2}}\right)=F\left(e^{-i \phi_{2}}\right)=0$. Let $u+i v=F\left(e^{i \phi}\right)(0<\phi<2 \pi)$. Since

$$
u+i v=\frac{\partial \arg f^{*}\left(e^{i \phi}\right)}{\partial \phi}-i \frac{\partial \log \left|f^{*}\left(e^{i \phi}\right)\right|}{\partial \phi}
$$

(any branch of $\arg f^{*}\left(e^{i \phi}\right), 0<\phi<2 \pi$, may be chosen), it follows from the boundary behavior of $F(z)$ that $f^{*}(K)$ is the set $\{w:|w|<M,-\alpha<\arg w<$ $\alpha\} \cup\{w:|w|<d, \alpha \leqslant \arg w \leqslant 2 \pi-\alpha\}$ for some $d, M$ and $\alpha$ where $\alpha=$ $\arg f^{*}\left(e^{-i \phi_{2}}\right)$. Since the power series for $f^{*}(z)$ has real coefficients, the domain $f(K)$ is symmetric with respect to the real axis.

We have

$$
f^{*}(z)=z \exp \left[\int_{0}^{z}[F(t)-1] t^{-1} d t\right]
$$

where $F(t)$ is defined by (1.1).

From the previous discussion of the boundary behavior of $F(z)$ it follows that for a fixed $d$ and $M$, an $a$ and $b$ will be determined by

$$
f^{*}(-1)=-\exp \left[\int_{0}^{-1}[F(t)-1] t^{-1} d t\right]=d
$$

and

$$
f^{*}(1)=\exp \left[\int_{0}^{1}[F(t)-1] t^{-1} d t\right]=M .
$$

II. Preliminary material. In this section we formulate the Julia variational formula and show how it can be utilized to produce variations used in studying Problems 1 and 2 of $\S I$.

Let $f(z)$ be in $\mathcal{S}$ and map $K$ onto a domain $D$ whose boundary contains a piecewise analytic curve $\Gamma$ which has left- and right-hand tangents at each point. Let $n(w)$ be the unit normal to $\Gamma$ at $w \in \Gamma$. Let $\phi(w)$ be a real-valued, continuous, piecewise differentiable function on $\Gamma$ which vanishes at the nonanalytic points of $\Gamma$. If $\varepsilon>0$ is sufficiently small, $\hat{w}=w+\varepsilon \phi(w) n(w)$ maps $\Gamma, 1-1$, onto a curve $\hat{\Gamma}$, and $\hat{\Gamma} \cup \partial \hat{D} / \Gamma$ is the boundary of a domain $\hat{D}$. Julia [7] proved that if $\hat{f}(z)$ maps $K, 1-1$, onto $\hat{D}$ with $\hat{f}(0)=0$, then $\hat{f}(z)$ is given by

$$
\hat{f}(z)=f(z)+\frac{\varepsilon z f^{\prime}(z)}{2 \pi i} \int_{\Gamma} \frac{\zeta+z}{\zeta-z} \frac{\phi(w) n(w)}{\left[\zeta f^{\prime}(\zeta)\right]^{2}} d w+o(\varepsilon),
$$

where $o(\varepsilon)$ denotes a term analytic in $z, z \in K$, such that $o(\varepsilon) / \varepsilon$ converges uniformly to 0 for $z$ in compact subsets of $K$. Here $w=f(\zeta)$, for $\zeta=e^{i \theta}$ on the arc of $|\zeta|=1$ that corresponds to $\Gamma$ under $f(\zeta)$.

Let 


$$
d \Psi=\frac{n(w) \phi(w) d w}{i\left[\zeta f^{\prime}(\zeta)\right]^{2}}=\frac{\phi(w) d \theta}{\left|\zeta f^{\prime}(\zeta)\right|} .
$$

Then $d \Psi$ is real and has the sign of $\phi(w)$,

$$
\hat{f}(z)=f(z)+\frac{\varepsilon z f^{\prime}(z)}{2 \pi} \int_{\Gamma} \frac{\zeta+z}{\zeta-z} d \Psi+o(\varepsilon)
$$

and

$$
\hat{f}^{\prime}(0)=f^{\prime}(0)+\frac{\varepsilon}{2 \pi} \int_{\Gamma} d \Psi+o(\varepsilon) .
$$

Thus, we have from (2.3) that

$$
\log [\hat{f}(z) / z]=\log [f(z) / z]+\frac{\varepsilon}{2 \pi} \int_{\Gamma} \frac{z f^{\prime}(z)}{f(z)} \frac{\zeta+z}{\zeta-z} d \Psi+o(\varepsilon) .
$$

Hence

$$
\operatorname{Re}\left\{\left[\log \frac{\hat{f}(z)}{z}\right]\right\}-\operatorname{Re}\left\{\left[\log \frac{f(z)}{z}\right]\right\}=\frac{\varepsilon}{2 \pi} \int_{\Gamma} \sigma(w) d \Psi+o(\varepsilon)
$$

where

$$
\sigma(w)=\operatorname{Re}\left\{\frac{z f^{\prime}(z)}{f(z)} \frac{\zeta+z}{\zeta-z}\right\},
$$

$w=f(\zeta)$ and $\zeta=e^{i \theta}$. Thus

$$
h(\zeta)=\frac{z f^{\prime}(z)}{f(z)} \frac{\zeta+z}{\zeta-z}
$$

maps $|\zeta|=1$ onto a circle for fixed $z,|z|<1$. Consequently $\operatorname{Re}\{h(\zeta)\}$ varies in a certain monotonic manner as $\zeta$ moves on $|\zeta|=1$, i.e., there exists two points $e^{i \theta_{0}}$ and $e^{i \theta_{1}}, 0<\theta_{0}<\theta_{1}<\theta_{0}+2 \pi$, on $|\zeta|=1$ separating $|\zeta|=1$ into two arcs $\gamma_{1}$ and $\gamma_{2}$ such that $\operatorname{Re}\{h(\zeta)\}$ decreases strictly as $\zeta$ moves on each of the two arcs $\gamma_{1}$ and $\gamma_{2}$ from $e^{i \theta_{0}}$ to $e^{i \theta_{1}}$. The functiol. $\operatorname{Re}\{h(\zeta)\}$ attains its extreme values at the points $e^{i \theta_{0}}$ and $e^{i \theta_{1}}$.

We will now outline the method in which equation (2.5) and the monotonicity of $h(\zeta)$ can be used to solve Problems 1 and 2. The basic idea is due to Krzyz [9].

First consider a domain with boundary $\Gamma$ containing three analytic arcs that are images of three arcs of $|\zeta|=1$ under $f(\zeta)$. It follows from the monotonic behavior of $\operatorname{Re}\{h(\zeta)\}$, that two of the three arcs, denoted by $l_{1}$ and $l_{2}$ satisfy

$$
\min _{w \in l_{1}} \sigma(w) \geqslant \max _{w \in l_{2}} \sigma(w) .
$$

In the sequel (2.7) will be referred to as the monotonic property of $\sigma(w)$. 
If we consider the two arcs $l_{1}$ and $l_{2}$ where (2.7) holds, we can choose a $\phi(w)>0$ and $\mathrm{a} \phi(w)<0$ on the open arcs $l_{1}$ and $l_{2}$, respectively, and $\phi(w)=0$ on the remainder of the boundary such that

$$
\int_{l_{1}} \frac{\phi(w) n(w)}{i\left[\zeta f^{\prime}(\zeta)\right]^{2}} d w=\int_{l_{2}} \frac{[-\phi(w)] n(w)}{i\left[\zeta f^{\prime}(\zeta)\right]^{2}} d w .
$$

Inequality (2.7), and (2.2), in view of the monotonicity property of $\sigma(w)$, imply

$$
\int_{l_{1}} \frac{\sigma(w) \phi(w) n(w)}{i\left[\zeta f^{\prime}(\zeta)\right]^{2}} d w>\int_{l_{2}} \frac{\sigma(w)[-\phi(w)] n(w)}{i\left[\zeta f^{\prime}(\zeta)\right]^{2}} d w .
$$

It follows from (2.4) that, when (2.8) holds, $\hat{f}^{\prime}(0)=f^{\prime}(0)$, and when (2.9) holds, $\operatorname{Re}\{\log (\hat{f}(z) / z)\}>\operatorname{Re}\{\log (f(z) / z)\}$, where $z \in K$ is fixed. If $\hat{f}(z)$ belongs to a given class of functions containing $f(z)$, and if such a construction is possible, the function $f(z)$ clearly cannot yield a solution to Problem 2 for the given class in the maximum case.

Similarly, putting $f_{1}(z)=-f(-z)$ we obtain a function $\hat{\hat{f}}(z)$ such that $\operatorname{Re}\{\log (\hat{f}(z) / z)\}<\operatorname{Re}\{\log (f(z) / z)\}$, where $z \in K$ is fixed. Thus whenever this construction is possible and $\hat{f}(z)$ belongs to a given class of functions containing $f(z)$, the function $f(z)$ evidently cannot yield a minimal value to the functional $\operatorname{Re}\{\log (f(z) / z)\}$ for the given class.

III. Boundary variations. Let $\mathscr{B}$ denote the class of all domains that are the range of functions in $\delta$ and whose boundaries lie in the closure of the annulus $S$. Let $\Omega \in \mathscr{B}$ with boundary $\Gamma$. We will show how to obtain a new domain in $B$ by considering certain combinations of the following three types of variations of a linear segment contained in $\Gamma$. Let $D A, A B$ and $B C$ be any three adjacent arcs of $\Gamma$, with $A B$ a straight line segment, and denote by $\gamma$ the remainder of $\Gamma$. We shall define:

VARIATION I. An inward rotation of $A B$ about an endpoint, say $A$, whenever $A B$ is not tangent to $\partial K_{d}$ and $B C$ is either

(a) a straight line segment, or

(b) an arc of $\partial K_{M}$ or $\partial K_{d}$.

VARIATION II. An outward rotation of $A B$ about an endpoint, say $A$, whenever either

(a) $B \notin \partial K_{M}$ or $\partial K_{d}$ and $B C$ is a straight line segment, or

(b) $B \in \partial K_{M}\left(\partial K_{d}\right)$ and $B C$ is either a straight line segment or an arc of $\partial K_{M}\left(\partial K_{d}\right)$.

To define Variation $\mathrm{I}(\mathrm{a})$ when $A B$ and $B C$ are straight sides we let $\lambda$ equal the interior angle at $A B C$ and consider four cases:
(i) $0<\lambda \leqslant \pi / 2$,
(ii) $\pi / 2<\lambda<\pi$,
(iii) $\pi<\lambda<3 \pi / 2$,
(iv) $3 \pi / 2<\lambda<2 \pi$. 
We rotate the side $A B$ inward by moving $B$ either along the side $B C$ to $B^{\prime} \in B C$ in cases (i) and (ii), or, along the extensions of the side $B C$ to $B^{\prime}$ on this extension in cases (iii) and (iv). In all four cases we then use the Julia variational formula (2.1) to obtain the mapping function, $f_{1}(z)$, for the domain $\Omega^{\prime}$, bounded by $\gamma D A B^{\prime} C$. Since $\Omega^{\prime}$ is not obtained from $\Omega$ by a normal displacement in all cases, we will need an intermediate step to obtain the desired variational formula for $f_{1}(z)$, i.e., we first make a normal displacement of $\partial \Omega$ obtaining a domain $\Omega^{\prime \prime}$, and the corresponding mapping function, $f_{2}(z)$ directly from $(2.1)$. We then show that the correction term $f_{1}(z)-f_{2}(z)$ is $O(\varepsilon)$ uniformly on compact subsets of $K$.

In case (i) we draw through $B$ the inward perpendicular $B B^{\prime \prime}$ to $A B$ where $B^{\prime \prime}$ is chosen such that $A B^{\prime \prime} \cap K_{d}$ is empty. The side $A B^{\prime \prime}$ meets $B C$ at $B^{\prime}$. In order to obtain a normal displacement we rotate side $B B^{\prime \prime}$ inward to $B B_{1}^{\prime \prime}$, where $B_{1}^{\prime \prime}$ lies on $A B^{\prime}$ and angle $A B B_{1}^{\prime \prime}$ is less than angle $A B B^{\prime}$. Denote angle $A B B_{1}^{\prime \prime}$ by $\beta$. Let $B_{1}$ be the perpendicular projection of $B_{1}^{\prime \prime}$ onto $A B$. Denote angle $B_{1} A B_{1}^{\prime \prime}$ by $\alpha$. We define

$$
\varepsilon \phi(w)= \begin{cases}-\varepsilon|w-A| \tan \alpha, & w \in A B_{1}, \\ -|w-B| \tan \beta, & w \in B_{1} B, \\ 0, & \text { otherwise. }\end{cases}
$$

Let $\Omega^{\prime \prime}$ be the varying domain defined by the normal displacement $\varepsilon \phi(w) n(w)$ of $\partial \Omega$. Let $f_{2}(z)$ be the corresponding mapping function such that $f_{2}(K)=\Omega^{\prime \prime}$. The Julia variational formula may be applied, giving

$$
f_{2}(z)-f(z)=\frac{\varepsilon z f^{\prime}(z)}{2 \pi i} \int_{A B} \frac{\zeta+z}{\zeta-z} \frac{\phi(w) n(w)}{\left[\zeta f^{\prime}(\zeta)\right]^{2}} d w+o(\varepsilon) .
$$

We denote by $\Omega^{\prime}$, the varying domain with boundary $\gamma D A B^{\prime} C$, and let $f_{1}(z)$ be its corresponding mapping function such that $f(K)=\Omega^{\prime}$. We will show

$$
f_{2}(z)-f_{1}(z)=o(\varepsilon) \text {. }
$$

Let $g_{2}\left(w, w_{0}\right)$ and $g_{1}\left(w, w_{0}\right)$ be the Green's functions for $\Omega^{\prime \prime}$ and $\Omega^{\prime}$, respectively. Then, since $\Omega^{\prime \prime}$ can be obtained by a suitable normal displacement of $\partial \Omega^{\prime}$ (using a finite number of steps if necessary) we have, using an argument similar to that of Krzyz [9], $g_{2}(w, 0)-g_{1}(w, 0)=o(\varepsilon)$. (3.4) then follows by a straightforward argument utilizing the fact that $g_{i}(w, 0)=$ $-\log \left|f_{i}^{-1}(w)\right|, i=1,2$.

Thus by comparing the corresponding mapping functions of $\Omega^{\prime}$ and $\Omega$ we have

$$
f_{1}(z)-f(z)=\frac{\varepsilon z f^{\prime}(z)}{2 \pi i} \int_{A B} \frac{\zeta+z}{\zeta-z} \frac{\phi(w) n(w)}{\left[\zeta f^{\prime}(\zeta)\right]^{2}} d w+o(\varepsilon)
$$

where $\phi(w)$ is defined by (3.2). 
In case (ii) we draw through $B$ the inward perpendicular $B B^{\prime \prime}$ to $A B$ where $B^{\prime \prime}$ is chosen such that $A B^{\prime \prime} \cap K_{d}$ is empty. The prolongation of $A B^{\prime \prime}$ meets $B C$ at $B^{\prime}$. In order to obtain a normal displacement we rotate $B B^{\prime \prime}$ inward to $B B_{1}^{\prime \prime}$ until the angle $B B_{1}^{\prime \prime} B^{\prime}$ is less than $\pi / 2$. Denote the angle $A B B_{1}^{\prime \prime}$ by $\beta$. Let $B_{1}$ be the perpendicular projection of $B_{1}^{\prime \prime}$ onto $A B$. Let $\alpha=$ angle $B_{1} A B_{1}^{\prime \prime}$. Then $\phi(w)$ is defined by (3.2) and $\Omega^{\prime \prime}, f_{2}(z), \Omega^{\prime}$, and $f_{1}(z)$ are also defined as in case (i). Equation (3.5) follows using the same argument as in case (i).

In case (iii) equation (3.5) can be obtained directly from (2.1) without the need of a correction term as in (3.4). Case (iv) follows similarly to case (ii).

We now define Variation $\mathrm{I}(\mathrm{b})$. The varied domain will be obtained by rotating the side $A B$ such that $B$ moves along either the $\operatorname{arc} B C$ of $\partial K_{M}$ to $B^{\prime} \in B C$ or the extension along $\partial K_{d}$ of an $\operatorname{arc} B C$ of $\partial K_{d}$ to $B^{\prime}$ on this extension. In either case, proceeding as in Variation I(a), the variable domain $\Omega^{\prime}$ arises by deleting from $\Omega$ the curvilinear triangle $A B B^{\prime}$. Then the derivation of equation (3.5) follows as in I(a) except that the straight line segment $B B^{\prime}$ is replaced by an arc $B B^{\prime}$ of $\partial K_{M}$ or $\partial K_{d}$.

REMARKS. 1. If $\Omega$ is a polygonal domain then Variation I(a) preserves the number of vertices of the boundary of $\Omega$.

2. If Variation $I$ is applied to a nonradial segment of the boundary of a starlike domain in $B$, the varied domain is again starlike.

To define Variation II(a), where $A B$ and $B C$ are straight line segments with $B \notin \partial K_{M}$ or $\partial K_{d}$, we consider the four cases of $\lambda=$ angle $A B C$ as in (3.1), but we denote these cases as (i'), (ii'), (iii'), and (iv'), respectively. We will rotate the side $A B$ outward by moving $B$ either along an extension of the side $B C$ to $B^{\prime}$ on this extension in cases (i') and (ii') or along the side $B C$ to $B^{\prime} \in B C$ in cases (iii') and (iv'). By using the two step procedure, as in Variation I, we show that equation (3.5) holds in this variation with $\phi(w)$ appropriately defined. We give the details of case (i'), noting its similarity to case (ii). We observe that case (ii'), case (iii'), and case (iv') are similar to case (iii), case (iv), and case (i) respectively.

In case (i') we draw $B B^{\prime \prime}$-the outward perpendicular to $A B$ at $B$-in such a way that the prolongation of $B C$ meets the prolongation of $B B^{\prime \prime}$ at $B^{\prime}$ where $B^{\prime}$ does not lie outside $\partial K_{M}$. Rotate $B B^{\prime}$ inward to $B B_{1}^{\prime \prime}$ until angle $B B_{1}^{\prime \prime} B^{\prime}$ is less than $\pi / 2$. Let $\beta$ be the angle $A B B_{1}^{\prime \prime}$ which is less than $\pi / 2$. Let $B_{1}$ be the perpendicular projection of $B_{1}^{\prime \prime}$ onto $A B$. Denote angle $B_{1} A B_{1}^{\prime \prime}$ by $\alpha$. Then, we can define

$$
\varepsilon \phi(w)= \begin{cases}\varepsilon|w-A| \tan \alpha, & w \in A B_{1}, \\ |w-B| \tan \beta, & w \in B_{1} B, \\ 0, & \text { otherwise. }\end{cases}
$$

The variable domain $\Omega^{\prime \prime}$ will be determined by the normal displacement $\varepsilon \phi(w) n(w)$ of the boundary of $\Omega$, whereas $\Omega^{\prime}$ is the varying domain with the 
boundary $\gamma D A B^{\prime} C$. Comparing the corresponding mapping functions for $\Omega$ and $\Omega^{\prime}$ we obtain as in the previous cases, equation (3.5).

We define Variation II(b) where the endpoint $B$ of side $A B$ is in $\partial K_{M}$ (or $\partial K_{d}$ ) and $B C$ is an arc of $\partial K_{M}$ (or $\partial K_{d}$ ) or a straight line segment.

If $B C$ is an arc of $\partial K_{M}\left(\partial K_{d}\right)$, then the argument proceeds as in case (ii') [(iii')], except that the line $B B^{\prime}$ is replaced by an arc $B B^{\prime}$ of $\partial K_{M}\left(\partial K_{d}\right)$. The variable domain $\Omega^{\prime}$ arises by adjoining to $\Omega$ the curvilinear triangle $A B B$; with variable domain $\Omega^{\prime}$ arises by adjoining to $\Omega$ the curvilinear triangle $A B B^{\prime}$; with variable $B^{\prime}$ on $\partial K_{M}\left(\partial K_{d}\right)$. If $B C$ is a straight line segment, it is clear that if we obtain the variable domain $\Omega^{\prime}$ by adjoining to $\Omega$ the curvilinear triangle $A B B^{\prime}$ with variable $B^{\prime}$ remaining on $\partial K_{M}\left(\partial K_{d}\right)$, the proof of (3.5) proceeds as in case (ii') [(iii')].

We will use combinations of the above described variations in the following manner.

LEMMA 3.1. Let $f(z)$ be in a given subclass $\mathcal{T}$ of $\mathcal{S}$. Let $\Gamma$ denote the boundary of $f(K)$, where $\Gamma$ is contained in the closure of the annulus $S=\{w$ : $d<|w|<M\}$. Suppose $\Gamma$ contains two line segments $l_{1}$ and $l_{2}$ that satisfy inequality (2.7). If $l_{1}$ can be rotated outward as in Variation II while $l_{2}$ is rotated inward, as in Variation I, in such a way that the new mapping function $\hat{f}(z)$ produced is also in $\mathcal{T}$, then the function $f(z)$ cannot give a solution to Problem 2 for the given class $\mathcal{T}$.

Proof. The proof follows from the discussion in §II and, in particular, inequality (2.9).

IV. Boundary variation of a slit mapping. In preparation for considering Problem 2 for the class $\delta_{d}^{*}(M)$ we will need to define a variation of a different type than those considered in \$III.

Suppose $\Omega=f(K), f \in \delta_{d}^{*}(M)$, and $\Gamma=\partial \Omega$ is a curvilinear polygon. Assume $\Gamma$ contains a linear slit of $\Omega$, i.e., a radial segment each "side" of which is a boundary arc of $\Omega$. Let $w_{0}=f\left(e^{i \theta_{0}}\right)$ be the tip of the slit $T$. Following Loewner [10] we define

VARIATION III. The shortening of a linear slit.

We can parametrize $T$ by $w=w(t), 0<t<t_{0}, w(0)=w_{0}$, and denote by $w=g(z, t), g(0, t)=0, g^{\prime}(0, t)>0$, the function that maps $K$ onto $\Omega=f(K)$ minus the segment $\left[w_{0}, w(t)\right]$. We denote the domain $g(K, t)$ by $\Omega_{t}$. It follows from Loewner's theory that under these conditions $w=g(z, t)=e^{t} z+\ldots$ satisfies the Loewner equation

$$
\frac{\partial g}{\partial t}=z \frac{1+k(t) z}{1-k(t) z} \frac{\partial g}{\partial z}
$$

where $g(z, 0)=f(z)$ and $k(t)$ is a continuous function with $|k(t)|=1$ and 
$\lambda(t)=1 / k(t)$ is the point on $|z|=1$ whose image under $g(z, t)$ is the tip $w(t)$ of the shortened slit.

In order to utilize Variation III in solving Problem 2 for $\delta_{d}^{*}(M)$ we will combine Variation III with Variation I of §III for functions $f \in \delta_{d}^{*}(M)$. We then show that if a certain relationship exists between the shortened slit and the rotated side, the function $f(z)$ cannot yield a solution to Problem 2 within $\delta_{d}^{*}(M)$.

First we note that since $\partial \Omega_{t}$ is a curvilinear polygon, $g(z, t)$ has a continuous extension to $|z|=1$.

Since $g(z, t)=e^{t} z+\ldots, g^{\prime}(0, t)>1$. To obtain a function that is normalized, we consider the following: If there exists a side $A B$ of $\partial \Omega_{t}$ that can be rotated inward as in Variation I of §III, then the Julia variational formula (2.1) defines a function $\hat{g}(z, t, \varepsilon)$ that maps $K$ onto a domain with the same boundary as $\Omega_{t}$ except that the side $A B$ has been rotated inward appropriately. For convenience we define $\phi(w)$ so that $\phi(w)>0$ on $A B$ and use $-\varepsilon$ for $\varepsilon>0$ in (3.5). We obtain from equation (3.5)

$$
\hat{g}(z, t, \varepsilon)=g(z, t)-\frac{\varepsilon z g^{\prime}(z, t)}{2 \pi} \int_{A B} \frac{\zeta+z}{\zeta-z} d \Psi+o(\varepsilon),
$$

where $g^{\prime}(z, t)=\partial g / \partial z$ and, as in (2.2),

$$
d \Psi=\phi(w) n(w) d w / i\left[\zeta g^{\prime}(\zeta)\right]^{2}
$$

is real and positive for $\phi(w)>0$. From (4.2) we obtain

$$
\hat{g}(z, t, \varepsilon)=e^{t}\left[1-\frac{\varepsilon}{2 \pi} \int_{A B} d \Psi+o(\varepsilon)\right] z+\ldots
$$

To normalize $\hat{g}$ we define $t=t(\varepsilon)$ by

$$
e^{t}\left[1-\frac{\varepsilon}{2 \pi} \int_{A B} d \Psi+o(\varepsilon)\right]=1
$$

i.e.,

$$
t=-\log \left[1-\frac{\varepsilon}{2 \pi} \int_{A B} d \Psi+o(\varepsilon)\right]
$$

It follows that

$$
\left.\frac{\partial t}{\partial \varepsilon}\right|_{e=0}=\frac{1}{2 \pi} \int_{A B} d \Psi .
$$

When $z=z_{0}$ in (2.6) we obtain

$$
\sigma_{1}(w)=\operatorname{Re}\left\{\frac{z_{0} f^{\prime}\left(z_{0}\right)}{f\left(z_{0}\right)} \frac{\zeta+z_{0}}{\zeta-z_{0}}\right\} .
$$

The main result of this section is

LEMMA 4.1. Let $f(z) \in \delta_{d}^{*}(M), f(K)=\Omega, \Gamma=\partial \Omega$, and $|f(z)|<\left|f\left(z_{0}\right)\right|$ 
for $|z|=r, 0<r<1$. Suppose $\Gamma$ contains a radial slit $T$ of $\Omega$. Let $l_{1}^{*}$ be the image under $f(z)$ of a sufficiently small arc, containing $e^{i \theta_{0}}$, of $|\zeta|=1$, where $f\left(e^{i \theta_{0}}\right)$ is the tip of $T$. Also assume $\Gamma$ contains a straight line segment $l_{2}$ such that $l_{1}^{*}$ and $l_{2}$ satisfy

$$
\min _{w \in l_{1}^{*}} \sigma_{1}(w)>\max _{w \in l_{2}} \sigma_{1}(w) .
$$

If the slit $T$ is shortened as in Variation III, while $l_{2}$ is rotated inward as in Variation I of §III in such a way that the new mapping function produced is also in $\delta_{d}^{*}(M)$, then the function $f(z)$ cannot yield a solution to Problem 2 for $\delta_{d}^{*}(M)$.

PROOF. If the variations are performed in such a way that the class $\delta_{d}^{*}(M)$ is preserved, we need only show that (4.6) implies that the function $\hat{g}$, as defined by (4.2), has a greater maximum (minimum) modulus on $|z|=r$ than $f(z)$. Let $z_{0},\left|z_{0}\right|=r$, be the point where the $\max (\min )$ of $|f(z)|$ occurs. Then when (4.6) holds, we determine the existence of an $\varepsilon_{0}>0$ such that

$$
\left|\hat{g}\left[z_{0}, t\left(\varepsilon_{0}\right)\right]\right|>f\left(z_{0}\right) \text {. }
$$

We do this by showing that (4.6) implies

$$
\left.\frac{\partial}{\partial \varepsilon}\left[\log \left|\frac{\hat{g}\left[z_{0}, t(\varepsilon)\right]}{z_{0}}\right|\right]\right|_{\varepsilon=0}>0 .
$$

(4.8) implies the existence of an $\varepsilon_{1}>0$ such that for all $\varepsilon \in\left[0, \varepsilon_{1}\right]$,

$$
\left|\hat{g}\left[z_{0}, t(\varepsilon)\right]\right|>\left|\hat{g}\left(z_{0}, 0\right)\right|=\left|f\left(z_{0}\right)\right| \text {. }
$$

Then, since $\hat{g}$ is normalized for any $\varepsilon$ satisfying (4.3) there exists a positive $\varepsilon_{0} \in\left(0, \varepsilon_{1}\right)$ such that (4.8) holds.

To prove (4.8), we have from (4.1), (4.2), and (4.4), that

$$
\begin{aligned}
\left.\frac{\partial}{\partial \varepsilon}\left[\log \left|\frac{\hat{g}\left[z_{0}, t(\varepsilon)\right]}{z_{0}}\right|\right]\right|_{\varepsilon=0} & \\
= & \operatorname{Re}\left\{\frac { 1 } { \hat { g } [ z _ { 0 } , t ( \varepsilon ) ] } \left[\frac{\partial g\left[z_{0}, t(\varepsilon)\right]}{\partial \varepsilon}\right.\right. \\
& \left.\left.-\frac{\partial}{\partial \varepsilon}\left(\frac{\varepsilon z_{0} g^{\prime}\left[z_{0}, t(\varepsilon)\right]}{2 \pi} \int_{A B} \frac{\zeta+z_{0}}{\zeta-z_{0}} d \Psi+o(\varepsilon)\right)\right]\right\}\left.\right|_{e=0}
\end{aligned}
$$




$$
\begin{gathered}
=\operatorname{Re}\left\{\frac { 1 } { \hat { g } } \left[\frac{\partial}{\partial t} g\left[z_{0}, t(\varepsilon)\right] \frac{\partial t}{\partial \varepsilon}\right.\right. \\
-\varepsilon \cdot \frac{\partial}{\partial t}\left(\frac{z_{0} g^{\prime}\left[z_{0}, t(\varepsilon)\right]}{2 \pi} \int_{A B} \frac{\zeta+z_{0}}{\zeta-z_{0}} d \Psi+o(1)\right) \cdot \frac{\partial t}{\partial \varepsilon} \\
\left.\left.-\frac{z_{0} g^{\prime}\left[z_{0}, t(\varepsilon)\right]}{2 \pi} \int_{A B} \frac{\zeta+z_{0}}{\zeta-z_{0}} d \Psi+o(1)\right]\right\}\left.\right|_{\varepsilon=0} \\
=\frac{z_{0} f^{\prime}\left(z_{0}\right)}{f\left(z_{0}\right)}\left[\frac{1}{2 \pi} \int_{A B} d \Psi \cdot \operatorname{Re}\left\{\frac{1+e^{-i \theta_{0} z_{0}}}{1-e^{-i \theta_{0}}}\right\}\right. \\
\left.\quad-\frac{1}{2 \pi} \int_{A B} \operatorname{Re}\left\{\frac{\zeta+z_{0}}{\zeta-z_{0}}\right\} d \Psi\right]
\end{gathered}
$$

provided we justify the existence of

$$
\begin{aligned}
& \frac{\partial}{\partial t}\left(\frac{z_{0} g^{\prime}\left[z_{0}, t(\varepsilon)\right]}{2 \pi} \int_{A B} \frac{\zeta+z_{0}}{\zeta-z_{0}} d \Psi\right) \\
& \quad=\frac{\partial}{\partial t}\left[\frac{z_{0} g^{\prime}\left[z_{0}, t(\varepsilon)\right]}{2 \pi} \int_{A B} \frac{\zeta+z_{0}}{\zeta-z_{0}} \frac{\phi(w) n(w) d w}{i\left[\zeta g^{\prime}(\zeta)\right]^{2}}\right] .
\end{aligned}
$$

We observe that (4.10) is positive if

$$
\operatorname{Re}\left\{\frac{z_{0} f^{\prime}\left(z_{0}\right)}{f\left(z_{0}\right)} \frac{\zeta_{0}+z_{0}}{\zeta_{0}-z_{0}}\right\} \int_{A B} d \Psi>\int_{A B} \operatorname{Re}\left\{\frac{z_{0} f^{\prime}\left(z_{0}\right)}{f\left(z_{0}\right)} \frac{\zeta+z_{0}}{\zeta-z_{0}}\right\} d \Psi
$$

where $\zeta_{0}=e^{i \theta_{0}}$ is the preimage of the tip of the slit $T$. Since $l_{2}$ and $l_{1}^{*}$, which contains the tip $f\left(e^{i \theta_{0}}\right)$ of $T$, satisfy (4.6), (4.12) holds, and (4.8) follows. Thus (4.7) will be proved when we justify the existence of (4.11). We first investigate the integral in (4.11). The existence of the partial of this integral presents no difficulty when $A$ and $B$ are interior points of a straight line segment in $\Gamma$. So, assume $\Gamma$ has a corner at $A$ and the section of $\Gamma$ adjacent to $A B$ at $A$ is either a straight line segment or an arc of $\partial K_{M}$. For the cases when $\phi(w)$ has different left and right derivatives at a point $B_{1}$ in the interior of $A B$ we will split the integral into the sum of two integrals, where one integral is taken over $A B_{1}$ and the other integral is taken over $B_{1} B$. It clearly suffices to justify the existence of the integral in (4.11) over $A B_{1}$. We use the fact [3] that in a neighborhood of a corner, say $g\left(\zeta_{1}, t\right)$, of the boundary of $\Omega_{t}$ we have

$$
g(\zeta, t)=g\left(\zeta_{1}, t\right)+\left(\zeta-\zeta_{1}\right)^{1-\beta} h(\zeta)
$$

where $(1-\beta) \pi$ is the interior angle at the corner and $h(\zeta)$ is analytic and nonzero in a neighborhood of $\zeta_{1}$. Thus, 


$$
\int_{A B} \frac{\zeta+z}{\zeta-z} \frac{\phi(w) n(w) d w}{i\left[\zeta g^{\prime}(\zeta)\right]^{2}}
$$

can be written with the change of variable $\zeta=\exp \left\{i \arg \left[\zeta_{1}+\left(\zeta_{2}-\zeta_{1}\right) s\right]\right\}$ as

$$
k \int_{0}^{1}\left[\frac{\zeta+z}{\zeta-z}\left|\frac{\left(\zeta-\zeta_{1}\right) h(\zeta)}{(1-\beta) h(\zeta)+\left(\zeta-\zeta_{1}\right) h^{\prime}(\zeta)}\right| \operatorname{Im}\left\{\frac{\zeta_{2}-\zeta_{1}}{\zeta_{1}+\left(\zeta_{2}-\zeta_{1}\right) s}\right\}\right] d s
$$

where $k$ is a constant relative to $t$ and $\zeta_{1}$ and $\zeta_{2}$ are the preimages of $A$ and $B_{1}$ respectively under $g(\zeta, t)$. We observe that $\zeta=g^{-1}(w)$ satisfies the differential equation

$$
\frac{\partial \zeta}{\partial t}=\zeta \frac{1+k(t) \zeta}{1-k(t) \zeta}
$$

Indeed

$$
\frac{\partial \zeta}{\partial t}=\frac{\partial}{\partial w} g^{-1}(w) \cdot \frac{\partial w}{\partial t}=\zeta \frac{1+k(t) \zeta}{1-k(t) \zeta} .
$$

Let $\zeta_{0}$ belong to a compact subarc of $|\zeta|=1$ that does not contain the points $k(t), 0<t<t_{0}$. For $z \in K$ arbitrarily close to $\zeta_{0}$ we have for $w=$ $g(z, t) \in \Omega_{t}$,

$$
\frac{\partial z}{\partial t}=\frac{\partial}{\partial w} g^{-1}(w) \cdot \frac{\partial w}{\partial t}=z \frac{1+k(t) z}{1-k(t) z} .
$$

If we denote the right-hand side of (4.15) by $R(t, \zeta, s)=R$, then it can easily be shown that $R$ is continuous and satisfies a Lipschitz condition with respect to $t$ and that $\partial R / \partial \zeta$ and $\partial R / \partial s$ exist and are continuous for all $s \in[0,1]$ and $\zeta$ in a sufficiently small neighborhood of $\zeta_{0}$. Hence by a standard theorem of differential equations [2], it follows that equation (4.15) is also valid for $w$ on a simple arc of $\partial \Omega_{t}$. Thus a straightforward calculation shows that the partial of the integrand of (4.13), with respect to $t$ exists and is continuous. Thereby we can conclude the existence of (4.11) upon showing that $\partial g^{\prime}(z, t) / \partial t$ exists. But, since the partial with respect to $z$ of the right-hand side of (4.1) exists and is continuous as a function of $z$ and $t$, the existence of $\partial g^{\prime}(z, t) / \partial t$ follows.

We observe that if $f(z)$ is rotated such that $|f(z)| \geqslant-f\left(-z_{0}\right)$, then, by the appropriate changes in the signs of the above arguments we have the existence of an $\varepsilon_{0}>0$ such that $\left|\hat{g}\left[-z_{0}, t\left(\varepsilon_{0}\right)\right]\right|<-f\left(-z_{0}\right)$, for an $l_{1}^{*}$ and $l_{2}$ satisfying (4.6). This completes the proof of Lemma 4.1.

In studying Problem 2 for $\varsigma_{d}^{*}(M)$ we shall also need to define two modifications of Variations I and II of §III. Using the same assumptions on a straight line segment $A B$ of $\Gamma=\partial f(K)$ as in §III we define 
VARIATION I'. The pushing in of a straight line segment $A B$ at the midpoint $C$ of $A B$, and

VARIATION II'. The pushing out of a straight line segment $A B$ at the midpoint $C$ of $A B$.

Variation $\mathrm{I}^{\prime}$ (II') is defined by rotating $A C$ and $B C$ inward (outward) as in Variation I (II) of §III, about $A$ and $B$ respectively. Both $A C$ and $C B$ are rotated $\alpha$ degrees, forming an isosceles triangle with base $A B$.

ReMARK. If Variation $\mathrm{I}^{\prime}$ or Variation $\mathrm{II}^{\prime}$ is applied to a nonradial segment of a starlike domain, and $\alpha$ is sufficiently small, the varied domain is again starlike.

Finally, we need to mention a special case of Variation I used by Hummel [5] and Robertson [13]. We define

VARIATION I". The hinging of one side of a slit at a point of the side.

Denote "one side" of a slit $T$ as $A B$ with $A$ at the tip and $B$ at the other end of the slit. Let $C \in A B, C \neq B$. Then the slit is hinged at $C$ if the straight line segment $C B$ is rotated inward as in Variation I of §III.

RemarKs. Assume that $f(z) \in \delta_{d}^{*}(M), \Gamma=\partial f(K)$, and that $\Gamma$ contains a radial slit $T$.

1. If $T$ is shortened as in Variation III, starlikeness is preserved.

2. If $\Gamma$ is a polygon then shortening $T$ also preserves the number of vertices of $\Gamma$.

3. If $T$ is hinged at a point of its side as in Variation $I^{\prime \prime}$, then starlikeness is preserved.

4. If $T$ is hinged at the tip of the slit using Variation I(a) of §III in Variation $I^{\prime}$, then the number of vertices of $\Gamma$ is preserved.

5 . We observe that both Variation $I^{\prime}$ and Variation $I^{\prime \prime}$ can be combined with Variation III to produce new domains in $\delta_{d}^{*}(M)$.

\section{Results for starlike functions.}

THEOREM 5.1. Let $f(z) \in \delta_{d}^{*}(M), \gamma(M) \leqslant d \leqslant 1$, and $M \geqslant 1$. Then, for $|z|=r$ fixed, $0<r<1,-f^{*}(-r) \leqslant|f(z)| \leqslant f^{*}(r)$, where

$$
f^{*}(z)=f^{*}(z, d, M)
$$

is defined by (1.2).

Proof. The proof of Theorem 5.1 consists of a series of lemmas.

Since the cases for $d=1$ and $M=1$ have already been discussed in $\S I$ we will assume $d<1$ and $M>1$.

Let $\mathscr{Q}$ denote the class of starlike domains $\Omega=f(K)$ for $f$ in $\delta_{d}^{*}(M)$. Let $\mathbb{Q}_{n}$ denote the subclass of $\mathcal{Q}$ which consists of all domains $\Omega$ whose boundary is an $n$-sided (linear) polygon. We allow radial slits in $\partial \Omega$ and we consider a slit as two "sides" of $\partial \Omega$. Clearly $\mathscr{Q}$ (and $\mathbb{Q}_{n}$ ) and the corresponding mapping 
functions form compact classes. Thus there exists a domain $\Omega_{1}^{*}=f^{*}(K)$ $\left[\Omega_{n}^{*}=f_{n}^{*}(K)\right]$ where $f^{*}(z)\left[f_{n}^{*}(z)\right]$ satisfies $|f(z)|<f^{*}\left(z_{0}\right)\left[\left|f_{n}(z)\right|<f_{n}^{*}\left(z_{0}\right)\right]$, $|z|=r<1$, for all $f(K) \in \mathbb{Q}\left[f_{n}(K) \in \mathbb{Q}_{n}\right]$.

The following lemma shows that $\cup \mathbb{Q}_{n}$ is dense in $\mathbb{Q}$.

LEMMA 5.2. If $\Omega \in \mathbb{Q}$, then there exists a sequence of domains $\left\{\Omega_{n}\right\}_{n=3}^{\infty}$ where $\Omega_{n} \in \mathbb{Q}_{n}$, such that $\Omega_{n}$ converges to $\Omega$, i.e., $\Omega$ is the kernel of the sequence $\left\{\Omega_{n}\right\}$ in the sense of Carathéodory.

Proof. It suffices to prove for every $\varepsilon>0$, there exists a polygonal domain $P_{n} \in \mathbb{Q}_{n}$ such that $\partial P_{n}$ is contained in an $\varepsilon$-neighborhood of $\partial \Omega$.

Let $\varepsilon>0$. Let $f(z) \in \delta_{d}^{*}(M)$ be the corresponding mapping function in $\delta_{d}^{*}(M)$ such that $f(K)=\Omega$. We consider $f_{r}(z)=f(r z) / r$. It follows from the properties of starlike functions and the maximum and minimum modulus principles that for $0<r<1, f_{r}(z) \in \delta_{d}^{*}(M)$ and $d<\left|f_{r}(z)\right|<M$ on $|z|=$ 1. So, for each $r, 0<r<1$, the image of $|z|=1$ under $f_{r}(z)$ is a $1-1$, analytic curve, contained in the annulus $S$ and bounds a starlike domain. Also, for $r$ sufficiently close to $1, \partial f_{r}(K)$ is contained in an $\varepsilon$-neighborhood of $\partial f(K)$. Let $r_{0}$ be sufficiently close to 1 and let $\Gamma=\partial f_{r_{0}}(K)$.

It is sufficient to prove that we can approximate $\Gamma$ to within $\varepsilon$ by a polygonal line $p$ such that the domain bounded by $p$ is starlike and has mapping radius one.

We divide the arc $\Gamma$ into two parts $\Gamma_{1}$ and $\Gamma_{2}$ such that $\Gamma_{1}$ consists of all the subarcs of $\Gamma$ where $\operatorname{Re}\left\{1+z f^{\prime \prime}(z) / f^{\prime}(z)\right\} \geqslant 0$ for $|z|=1$ and $\Gamma_{2}$ consists of all the subarcs of $\Gamma$ where $\operatorname{Re}\left\{1+z f^{\prime \prime}(z) / f^{\prime}(z)\right\}<0$, for $|z|=1$. It is easily shown that each of $\Gamma_{1}$ and $\Gamma_{2}$ contains only a finite number of components. Thus we need only show that each component can be replaced by an appropriate polygonal line.

Let $\gamma_{1}$ be a component of $\Gamma_{1}$ and $\gamma_{2}$ a component of $\Gamma_{2}$. We can approximate $\gamma_{1}\left(\gamma_{2}\right)$ from within (without) by connecting points on $\gamma_{1}\left(\gamma_{2}\right)$ and from without (within) by intersecting segments of tangents to neighboring points on $\gamma_{1}\left(\gamma_{2}\right)$. By taking points on $\gamma_{1}\left(\gamma_{2}\right)$ sufficiently close it follows that both methods of approximation produce a polygonal line $p$ having the same endpoints as $\gamma_{1}\left(\gamma_{2}\right)$ and such that $p$ is contained in an $\varepsilon$-neighborhood of $\gamma_{1}$ $\left(\gamma_{2}\right)$. Also, because of the nature of the methods of approximation, the domain bounded by $p+\Gamma \backslash \gamma_{1}\left(\gamma_{2}\right)$ is starlike.

Hence it suffices to prove that by combining the two methods of approximation the mapping radius can be preserved. Let $\gamma$ be an arbitrary component of either $\Gamma_{1}$ or $\Gamma_{2}$. We divide $\gamma$ into two proper subarcs, say $\gamma_{3}$ and $\gamma_{4}$. Then we approximate $\gamma_{3}$ from within by a polygonal line $p_{3}$ and we approximate $\gamma_{4}$ from without by a polygonal line $p_{4}$. If we denote the polygonal line $p_{3}+p_{4}$ by $p$, then using the fact that a refinement of either approximation appropriately increases or decreases the mapping radius, it follows that $p$ 
can be chosen so that the domain bounded by $p+\Gamma \backslash \gamma$ has mapping radius one. Thus, Lemma 5.2 is proved.

If $\Omega_{n}$ converges to $\Omega$, the Carathéodory Kernel Theorem assures that for the function $f_{n}$ and $f, f_{n}(K)=\Omega_{n}$ and $f(K)=\Omega, f_{n}(z)$ converges to $f(z)$ uniformly on compact subsets of $K$. Thus, it follows that any convergent subsequence $\left\{\Omega_{n_{k}}\right\}_{k=1}^{\infty}$ of the extremal domains for Problem 2 in $Q_{n_{k}}$ converges to a domain $\Omega_{1}^{*} \in \mathbb{Q}$, which is an extremal domain for Problem 2 within $\mathcal{Q}$. In order to characterize $\Omega_{1}^{*}$ we must first determine certain properties of $\partial \Omega_{n}^{*}$.

Lemma 5.3. Let $\Gamma_{n}^{*}$ be the boundary of the extremal domain $\Omega_{n}^{*}$ for Problem 2 within $\mathbb{Q}_{n} . \Gamma_{n}^{*}$ has at most two radial slits, and all but at most two of the distinct sides (not on slits) of $\Gamma_{n}^{*}$ are either chords of $\partial K_{M}$ or tangent to $\partial K_{d}$.

Proof. To show that $\Gamma_{n}^{*}=\partial \Omega_{n}^{*}$ has not more than two radial slits, we assume that $\Gamma_{n}^{*}$ has at least three radial slits. Since $f(z)$ has a continuous extension to $\partial K, \sigma_{1}(w)$ can be defined for all $w \in \Gamma_{n}^{*}$. Thus, recalling the monotonic property of $\sigma_{1}(w)$, as defined in (4.5), we let $M$ and $m$ denote the two points in $\Gamma_{n}^{*}$ where $\sigma_{1}(w)$ attains its maximum and minimum values, respectively. $M$ and $m$ divide $\Gamma_{n}^{*}$ into two arcs such that $\sigma_{1}(w)$ is strictly increasing as $w$ moves along either arc of $\Gamma_{n}^{*}$ from $m$ to $M$. At least one of these two arcs contains a radial slit and "a side" of another slit. Clearly, we can choose one of the slits, denoted by $T$, to define an $l_{1}^{*}$ and the "side", denoted by $A B$, of another slit, which we denote by $T^{\prime}$, with tip at $A$, to act as an $l_{2}$ in inequality (4.6). Recall, if $f\left(e^{i \theta_{0}}\right)$ is the tip of $T$, then $l^{*}$ is the image of a sufficiently small arc, containing $e^{i \theta_{0}}$, of $|\zeta|=1$. Now, since we can shorten the slit $T$ as in Variation III, of $\S \mathrm{IV}$, and hinge the side $A B$ of slit $T^{\prime}$ at $A$ as in Variation I" of $\S I V$, preserving starlikeness and the number of vertices in the boundary, we can produce another domain in $\mathbb{Q}_{n}$. Thus, Lemma 4.1 implies that $\Omega_{n}^{*}$ cannot be an extremal domain for Problem 2 within $\mathbb{Q}_{n}$. Since this yields a contradiction, $\Gamma_{n}^{*}$ can have no more than two radial slits.

Next, we assume that $\Gamma_{n}^{*}$ has at least three distinct sides (not sides of slits) which are neither chords of $\partial K_{M}$ nor tangent to $\partial K_{d}$. From monotonicity of $\sigma(w)$, one of the sides will act as an $l_{1}$ in inequality (2.7), and another side will act as an $l_{2}$ in (2.7). Since $l_{1}$ is not a chord of $\partial K_{M}$, it can be rotated outward, as in Variation II(a) of §III, about one of its endpoints, while preserving starlikeness and without increasing the number of vertices of the boundary. Since $l_{2}$ is not tangent to $\partial K_{d}$, it can be rotated inward as in Variation I(a) of \$III, preserving starlikeness and the number of vertices of the boundary. Hence, by rotating $l_{1}$ outward while rotating $l_{2}$ inward and taking $\phi(w)$ so that equality holds in (2.8), the new domain obtained will be in $\mathbb{Q}_{n}$. Thus, by Lemma 3.1, $\Omega_{n}^{*}$ cannot be an extremal domain for Problem 2 within $\mathbb{Q}_{n}$. This yields a contradiction, and Lemma 5.3 is proved.

We shall need 
LeMma 5.4. Let $R=\left\{w: d<r_{2}<|w|<r_{1}<M\right\}$. If $\Gamma_{n}^{*}=\partial \Omega_{n}^{*}$, then there exists, independently of $n$, a maximum number $N$ of sides of $\Gamma_{n}^{*}$ that intersect $\bar{R}$.

Proof. Let $C_{1}=\partial K_{r_{1}}$ and $C_{2}=\partial K_{r_{2}}$.

We first consider those sides of $\partial \Omega_{n}^{*}$ that are chords of $\partial K_{M}$. If a chord of $\partial K_{M}$ intersects $\bar{R}$, then there exists a minor arc of $\partial K_{M}$ of fixed minimum arc length, say $c_{1}$, subtended by the chord. The minimum arc length $c_{1}$ would be the arc length of the minor arc subtended by a chord of $\partial K_{M}$ which is tangent to $C_{1}$. Choose an integer $k_{1}$ such that $k_{1} c_{1}$ exceeds the circumference of $\partial K_{M}$. Then, independently of $n$, no more than $k_{1}$ sides of $\partial \Omega_{n}^{*}$, which are chords of $\partial K_{M}$, can intersect $\bar{R}$.

If a $\partial K_{d}$-tangent, say $l$, intersects $\bar{R}$ we let $P$ be the point which is the radial projection of a point where $l$ intersects the circle $C_{2}$. Let $P^{\prime}$ be the point of tangency of the $\partial K_{d}$-tangent $l$. The arc length of $P P^{\prime}$ is a fixed length $c_{2}$, depending only on $\partial K_{d}$ and $C_{2}$. Since starlikeness prevents overlapping of the radial projections of the $\partial K_{d}$-tangents on $\partial K_{d}$, if $k_{2}$ is an integer such that $k_{2} c_{2}$ exceeds the circumference of $\partial K_{d}$, then there can be no more than $k_{2}$ $\partial K_{d}$-tangents that intersect $\bar{R}$.

Allowing the possibility of two radial slits and two sides of $\partial \Omega_{n}^{*}$ that are not $\partial K_{d}$-tangents or chords of $\partial K_{M}$, it follows that no more than $N=k_{1}+k_{2}+$ 6 sides of $\partial \Omega_{n}^{*}$, independent of $n$, can intersect $R$, and Lemma 5.4 is proved. We now begin the characterization of $\Omega_{1}^{*}$.

LEMMA 5.5. If $\Gamma_{1}^{*}=\partial \Omega^{*}$, then $\Gamma_{1}^{*}$ has at most four radial slits.

Proof. We shall need the following property, denoted by $P_{1}$, of the kernel $\Omega$ of a sequence of domains $\left\{\Omega_{n}\right\}_{n=1}^{\infty}$. Let $C$ be a compact set contained in $\partial \Omega$. For every $\delta>0$, there exists an $N=N(\delta)$ such that for each $n>N$, if $w \in C$, then there exists a $w_{n} \in \partial \Omega_{n}$ such that $\left|w_{n}-w\right|<\delta$. This property follows easily from the definition of the kernel of a sequence of domains.

Now, assume $\Gamma_{1}^{*}$ has at least five radial slits. We draw two circles $C_{1}$ and $C_{2}$, centered at the origin with radii $r_{1}$ and $r_{2}$, respectively. $r_{1}$ and $r_{2}$ are determined by requiring that the two points of the intersection of $C_{1}$ and $C_{2}$ with one of the shortest of five of the radial slits, divides that slit into thirds. Let $\left\{a_{1}, a_{2}, \ldots, a_{5}\right\},\left\{b_{1}, b_{2}, \ldots, b_{5}\right\}$ be the points of intersection of these slits with $C_{1}$ and $C_{2}$ respectively, where $a_{i}$ and $b_{i}$ are on the same slit, $i=1$, $2, \ldots, 5$. Choose $c_{i}$ the midpoint of $\left[a_{i}, b_{i}\right]$. Choose $\delta$ so small that no chord or $\partial K_{d}$-tangent passing through a $\delta$-neighborhood of an $a_{i}$ or $b_{i}$ passes through a $\delta$-neighborhood of $c_{i}$ for $i=1,2, \ldots, 5$. Let $C$ be the set of segments $\left\{\left[a_{i}, b_{i}\right] \mid 1 \leqslant i<5\right\}$. Then Property $\mathrm{P}_{1}$ assures that for this $\delta$ there exists an $N$ such that a point of $\partial \Omega_{n}^{*}$ lies within a $\delta$-neighborhood of each point of $C$. From starlikeness, the only way the boundary, $\partial \Omega_{N}^{*}$, can intersect a $\delta$-neighborhood of $a_{i}$, a $\delta$-neighborhood of $b_{i}$ and a $\delta$-neighborhood of $c_{i}$ is 
for $\partial \Omega_{N}^{*}$ to contain at least one side which is neither a chord of $\partial K_{\cdot M}$ nor a $\partial K_{d}$-tangent for each $i=1,2, \ldots, 5$. From the definition of $\delta$ this would require $\partial \Omega_{N}^{*}$ to have more than two radial slits or more than two distinct sides which are neither chords of $\partial K_{M}$ nor $\partial K_{d}$-tangents. But this implies a contradiction to Lemma 5.3 and Lemma 5.5 is proved.

LEMMA 5.6. Let $\Gamma_{1}^{*}=\partial \Omega_{1}^{*}$. Then the intersection of $\Gamma_{1}^{*}$ with any proper subannulus of $S$ is comprised of a finite number of linear segments.

Proof. Let $R=\left\{w: d<r_{2}<|w|<r_{1}<M\right\}$ be a proper subannulus of $S$. We show that $R \cap \Gamma_{1}^{*}$, is comprised of linear segments for any $r_{1}, r_{2}$, $d<r_{2}<r_{1}<M$. If $\Gamma_{n}^{*}=\partial \Omega_{n}^{*}$ then, from Lemma 5.4 for each $n, \bar{R} \cap \Gamma_{n}^{*}$ is a set of at most $N=N(\bar{R})$ line segments, i.e., $\bar{R} \cap \Gamma_{n}^{*}=\left\{\left[a_{n 1}, b_{n 1}\right],\left[a_{n 2}\right.\right.$, $\left.\left.b_{n 2}\right], \ldots,\left[a_{n N}, b_{n N}\right]\right\}$. For an appropriate subsequence $\left\{n_{k}\right\}, a_{n_{k} i}$ and $b_{n_{k} i}$ converge to $a_{i}$ and $b_{i}$, respectively, for $i=1,2, \ldots, N$. Hence each point of the line segment $\left[a_{n_{k} i}, b_{n_{k} i}\right]$ converges to a corresponding point of the segment $\left[a_{i}, b_{i}\right]$ and $\left[a_{i}, b_{i}\right] \subset \bar{R}$. Also $\Omega_{n}^{*}$ converges to $\Omega_{1}^{*}$. Since each boundary point of $\Omega_{1}^{*}$ is a limit point of boundary points of the $\Omega_{n_{k}}^{*}$, it follows that $R \cap \Gamma_{1}^{*}$ is contained in the linear segments $\left\{\left[a_{i}, b_{i}\right] \mid 1 \leqslant i \leqslant N\right\}$ and Lemma 5.6 is proved.

LEMMA 5.7. $\Gamma_{1}^{*}=\partial \Omega_{1}^{*}$ contains no nonradial straight line segments in $S$ and at most two distinct radial sides (not on slits) in $S$.

Proof. Assume $A B$ is a nonradial straight line segment of $\Gamma_{1}^{*}$. Let $A_{1} B_{1}$, $A_{2} B_{2}, A_{3} B_{3}$, be three distinct closed line segments contained in $A B$ such that $A_{i} B_{i} \cap \bar{K}_{d}=\varnothing$ and $A_{i}$ and $B_{i}$ are distinct from $A$ and $B$ for $i=1,2.3$. Then from the monotone property of $\sigma(w)$ in (2.6), one of these subsets of $A B$, say $A_{1} B_{1}$, will act as in $l_{1}$ in inequality (2.7), while another of these subsets of $A B$, say $A_{2} B_{2}$ will act as an $l_{2}$ in (2.7). We can now push out $l_{1}=A_{1} B_{1}$ at its midpoint as in Variation II' of $\S I V$, and push in $l_{2}=A_{2} B_{2}$ at its midpoint as in Variation $\mathrm{I}^{\prime}$ of $\S \mathrm{IV}$, taking $\phi(w)$ so that equality holds in (2.8). Then, since $A B$ is nonradial, these variations preserve starlikeness and the varied domain, thus obtained, is in $Q$. Hence, by Lemma $3.1, \Omega_{1}^{*}$ cannot be extremal for Problem 2 within $Q$. Since this implies a contradiction, $\Gamma_{1}^{*}$ contains no nonradial straight line segments in $S$.

Thus, $\Gamma_{1}^{*}$ contains only radial sides connecting points of $\partial K_{d}$ to $\partial K_{M}$, radial slits, and arcs of $\partial K_{d}$ and $\partial K_{M}$.

We next assume that $\Gamma_{1}^{*}$ contains at least three radial sides, not on slits. From the monotonicity property of $\sigma(w)$, one of the radial sides will act as an $l_{1}$ in inequality (2.7) while another of these radial sides will act as an $l_{2}$ in (2.7). It is clear that if $l_{1}$ is rotated outward, as in Variation II, of §III, about its endpoint on $\partial K_{M}$ while $l_{2}$ is rotated inward, as in Variation I, about its endpoint on $\partial K_{d}$, while taking $\phi(w)$ so that equality holds in $(2.8)$, then 
starlikeness is preserved and the new domain obtained is in $\mathcal{Q}$. Since this implies a contradiction, Lemma 5.7 is proved.

LEMMA 5.8. $f^{*}(z, d, M)$, defined by (1.2), is an extremal function for Problem 2 for the class $\mathcal{S}_{d}^{*}(M)$.

Proof. Let $\Omega_{1}^{*}$ be the extremal domain of Lemma 5.5 and Lemma 5.7. We show that $\Omega_{1}^{*}=e^{i \alpha f^{*}}(K)$, from which the lemma follows. From the previous lemmas $\Gamma_{1}^{*}=\partial \Omega_{1}^{*}$ is reduced to having at most two distinct radial sides, not on slits, the connecting arcs of $\partial K_{d}$ and $\partial K_{M}$ and at most four radial slits. Thus we need only show that $\Gamma_{1}^{*}$ cannot have any radial slits unless $\Omega_{1}^{*}$ is a rotation of $K_{M} \backslash T_{1}$ where $T_{1}$ is the radial slit from $-M$ to $-d$. [Note that in this case $d=\gamma(M)$.] We can assume $\left|f^{*}(z)\right| \leqslant f^{*}\left(r e^{i \theta_{0}}\right)$ where $f^{*}(z) \in$ $\mathcal{S}_{d}^{*}(M)$ and $f^{*}(K)=\Omega_{1}^{*}$. To obtain a contradiction we shall assume $\Gamma_{1}^{*}$ has a radial slit $T$ and $\Omega_{1}^{*}$ is not a rotation of $K_{M} \backslash T_{1}$. Since m.r. $\Omega_{1}^{*}=1$, a routine mapping radius argument shows that either $\Gamma_{1}^{*}$ contains at least two radial slits or $K_{M} \backslash \overline{\Omega_{1}^{*}} \neq \varnothing$. We note, from Lemma 5.6, that if $K_{M} \backslash \overline{\Omega_{1}^{*}} \neq \varnothing$ then $\Gamma_{1}^{*}$ contains at least one straight side. Let $m$ be the point where the minimum value of $\sigma_{1}(w)$, as defined in (4.5), is attained. We consider two cases: (i) when $m \in T$ and (ii) when $m \notin T$.

For case (i), if there exists a distinct straight side (i.e. not a radial slit) say $C D$, of $\Gamma_{1}^{*}$, then $C D$ would act as an $l_{1}$ in inequality (2.7) while one of the "sides", say $A B$, of the slit $T$, with $A$ at the tip, would act as an $l_{2}$ in inequality (2.7). We can now rotate $C D=l_{1}$ outward, as in Variation II of $\S I I I$, about an endpoint while hinging side $A B$ at $A$, as in Variation $\mathrm{I}^{\prime}$, of $\S \mathrm{IV}$, and taking $\phi(w)$ so that equation (2.8) holds, which yields a domain with mapping radius one. Also, these variations can be performed in such a way as to preserve starlikeness, and the boundary of the new domain obtained remains in the closure of the annulus $S$. Thus, by Lemma 3.1, $\Omega_{1}^{*}$ cannot be an extremal domain for Problem 2 within $\mathbb{Q}$. This contradicts the assumption that $\Omega_{1}^{*}$ is extremal.

For case (i), where $m \in T$, if there does not exist a distinct side of $\Gamma_{1}^{*}$, then there exists another radial slit, which we denote as $T^{\prime}$. It is clear from the monotonicity of $\sigma_{1}(w)$, that "one side", say $A B$, of $T$ with tip at $A$, can act as an $l_{2}$ in inequality (4.6), while a neighborhood, within $\Gamma_{1}^{*}$, of the tip, say $f^{*}\left(e^{i \theta_{0}}\right)$, of $T^{\prime}$ can act as an $l_{1}^{*}$ in (4.6); i.e., where $l_{1}^{*}$ is the image under $f^{*}(z)$ of a sufficiently small arc, containing $e^{i \theta_{0}}$, of $|\zeta|=1$. Then we can shorten the slit $T^{\prime}$, as in Variation III of $\S \mathrm{IV}$, and hinge the side $A B$ of slit $T$, as in Variation $I^{\prime}$, to produce a new domain in $\mathcal{Q}$. By Lemma $4.1, \Omega_{1}^{*}$ cannot be an extremal domain for Problem 2 within $Q$, which implies a contradiction.

For case (ii), where $m \notin T$, it follows that, as $w$ moves around $\Gamma_{i}^{*}$ a sufficiently small neighborhood, within $\Gamma_{1}^{*}$, of the tip of the slit $T$, can act as an $l_{1}^{*}$ in inequality (4.6), while "one side", say $A B$, of $T$, with $A$ at the tip, has 
a point $C$ in $A B$ such that the straight line segment $C B$ can act as an $l_{2}$ in (4.6). Then we can shorten the slit $T$ and hinge the side $A B$ of the slit $T$ at $C$ to produce a new domain in $\mathbb{Q}$. Again by Lemma 4.1, this implies a contradiction.

Thus we have proved that $\Gamma_{1}^{*}$ consists of a single arc $D C$ of $\partial K_{M}$, two radial sides $A D$ and $B C$ [the sides of a slit when $d=\gamma(M)$ ] and the arc $A B$ (point) of $\partial K_{d}$, i.e., $\Omega_{1}^{*}=e^{i \alpha_{f}} f(K)$ where $f^{*}(z)=f^{*}(z, d, M)$ and Lemma 5.8 is proved.

If we take $\phi(w)$ positive on those parts of the boundary where $\sigma(w)\left[\sigma_{1}(w)\right]$ is small and negative where $\sigma(w)\left[\sigma_{1}(w)\right]$ is large, i.e., interchanging the roles of $l_{1}\left(l_{1}^{*}\right)$ and $l_{2}$, then an analogous argument proves that the same domain $f^{*}(K)$ also gives the least minimum modulus for all $f(z)$ in $\delta_{d}^{*}(M)$.

Thus, the function $f^{*}(z, d, M)$, as defined by (1.2), is an extremal function giving the sharp upper and lower bounds for $|f(z)|$, within $\delta_{d}^{*}(M),|z|=r$ fixed, $0<r<1$. Since $f^{*}(z, d, M)$ is a circular symmetric function [6] with respect to the positive real axis, the function, $\left|f^{*}(z, d, M)\right|$, attains its maximum and minimum values for $z=r,-r$ respectively. Therefore, Theorem 5.1 is proved.

THEOREM 5.9. If $f(z) \in \delta_{d}^{*}(M), \gamma(M)<d<1,1<M<\infty$ and $|z|=r$,

$$
0<r<\frac{1}{2}(3-\sqrt{5})
$$

then, for $f^{*}(z)=f^{*}(z, d, M)$, as defined in $\S \mathrm{I}$,

$$
\min _{|z|=r}\left|f^{* \prime}(z)\right|<\left|f^{\prime}(z)\right|<\max _{|z|=r}\left|f^{* \prime}(z)\right| \text {. }
$$

Proof. As in Theorem 5.1 it is only necessary to prove the result for the functional $\operatorname{Re}\left\{\log f^{\prime}(z)\right\}$. We use the method of proof of Theorem 5.1. It is clear that Theorem 5.9 will follow upon showing that the function obtained from $f^{\prime}(z)$ corresponding to the $\sigma(w)$ in (2.6) and $\sigma_{1}(w)$ in (4.6) has the same monotonic behavior as $\sigma(w)$ for $r$ satisfying (5.10).

We first proceed as in §II. In this proof, all the integrals are taken over the boundary $\Gamma$ and $d \Psi$ is defined as in (2.2). We shall assume that $z_{0}$ is such that $\operatorname{Re}\left\{\log f^{\prime}(z)\right\}<\operatorname{Re}\left\{\log f^{\prime}\left(z_{0}\right)\right\}$. Thus, using known estimates on functions in $\mathcal{S}^{*}$ it follows that $A\left(z_{0}\right)=1+z_{0} f^{\prime \prime}\left(z_{0}\right) / f^{\prime}\left(z_{0}\right)$ satisfies

$$
\frac{1-4\left|z_{0}\right|+\left|z_{0}\right|^{2}}{1-\left|z_{0}\right|^{2}} \leqslant A\left(z_{0}\right) \leqslant \frac{1+4\left|z_{0}\right|+\left|z_{0}\right|^{2}}{1-\left|z_{0}\right|^{2}} .
$$

We have from equation (2.1) that

$$
\hat{f}(z)=f(z)\left[1+\frac{\varepsilon}{2 \pi} \int \frac{z f^{\prime}(z)}{f(z)} \frac{\zeta+z}{\zeta-z} d \Psi\right]+o(\varepsilon) .
$$

Hence, by taking the derivative in (5.12), we obtain 


$$
\begin{aligned}
\hat{f}^{\prime}(z)=f^{\prime}(z)\left\{1+\frac{\varepsilon}{2 \pi} \int[\right. & \frac{2 \zeta z}{(\zeta-z)^{2}} \\
& \left.\left.+\left(1+\frac{z f^{\prime \prime}(z)}{f^{\prime}(z)}\right) \frac{\zeta+z}{\zeta-z}\right] d \Psi\right\}+o(\varepsilon) .
\end{aligned}
$$

So

$$
\begin{aligned}
\log \hat{f}^{\prime}(z)= & \log f^{\prime}(z) \\
& +\frac{\varepsilon}{2 \pi} \int\left[\frac{2 \zeta z}{(\zeta-z)^{2}}+A(z) \frac{\zeta+z}{\zeta-z}\right] d \Psi+o(\varepsilon),
\end{aligned}
$$

where $A(z)=1+z f^{\prime \prime}(z) / f^{\prime}(z)$. Taking real parts we obtain

where

$$
\operatorname{Re}\left\{\log \hat{f}^{\prime}(z)\right\}-\operatorname{Re}\left\{\log f^{\prime}(z)\right\}=\frac{\varepsilon}{2 \pi} \int \lambda(\zeta) d \Psi+o(\varepsilon)
$$

$$
\lambda(\zeta)=\operatorname{Re}\left\{\frac{2 \zeta z}{(\zeta-z)^{2}}+A(z) \frac{\zeta+z}{\zeta-z}\right\}
$$

If we define

$$
F(x)=\frac{2 x}{(1-x)^{2}}+A\left(z_{0}\right) \frac{1+x}{1-x}=A+2(1+A) x+\ldots,
$$

where $x=\bar{\zeta}_{0}$ in (5.13), then $F(x)$ defines an analytic function of $x \in K$. Using (5.11) a straightforward calculation shows that $\operatorname{Re}\left\{1+x F^{\prime \prime}(x) / F^{\prime}(x)\right\}$ $>0$ for $0<|x|=\left|\bar{\xi}_{0}\right|=r<\frac{1}{2}(3-\sqrt{5})$. Thus, for $r$ satisfying (5.10), $F(x)$ maps $|x|=r$ onto a convex curve. Consequently $\lambda(\zeta)$ has the same monotonic property as $\sigma[w(\zeta)]$ in (2.6).

Now we proceed as in \$IV. From equation (4.2) we have

$$
\begin{aligned}
& \hat{g}^{\prime}(z, t, \varepsilon) \\
& =g^{\prime}(z, t)\left\{1-\frac{\varepsilon}{2 \pi} \int\left[\frac{2 \zeta z}{(\zeta-z)^{2}}+\left(1+\frac{z g^{\prime \prime}(z, t)}{g^{\prime}(z, t)}\right) \frac{\zeta+z}{\zeta-z}\right] d \Psi\right\}+o(\varepsilon) .
\end{aligned}
$$

Thus

$$
\begin{aligned}
\operatorname{Re}\left\{\log \hat{g}^{\prime}(z, t, \varepsilon)\right\} & \\
= & \operatorname{Re}\left\{\log g^{\prime}(z, t)\right\} \\
& -\frac{\varepsilon}{2 \pi} \int \operatorname{Re}\left\{\frac{2 \zeta z}{(\zeta-z)^{2}}+\left(1+\frac{z g^{\prime \prime}(z, t)}{g^{\prime}(z, t)}\right) \frac{\zeta+z}{\zeta-z}\right\} d \Psi+o(\varepsilon) \\
= & \operatorname{Re}\left\{\log g^{\prime}(z, t)\right\}-\frac{\varepsilon}{2 \pi} \int \lambda(\zeta) d \Psi+o(\varepsilon) .
\end{aligned}
$$

Hence 


$$
\begin{aligned}
& \left.\frac{\partial}{\partial \varepsilon}\left[\log \left|\hat{g}^{\prime}(z, t, \varepsilon)\right|\right]\right|_{\varepsilon=0} \\
& =\left\{\operatorname{Re}\left[\frac{\partial^{2} g(z, t)}{\partial \varepsilon \partial z} g^{\prime}(z, t)\right]-\varepsilon \cdot \frac{\partial}{\partial \varepsilon}\left[\frac{1}{2 \pi} \int \lambda(\zeta) d \Psi+o(1)\right]\right. \\
& \left.=\left.\operatorname{Re}\left\{\frac{\partial}{\partial z} \frac{\partial g}{\partial t} \cdot \frac{\partial t}{\partial \varepsilon} / g^{\prime}(z, t)\right\}\right|_{\varepsilon=0}-\frac{1}{2 \pi} \int \lambda(\zeta) d \Psi+o(1)\right\}\left.\right|_{\varepsilon=0} \\
& =\operatorname{Re}\left\{\frac{2 \zeta_{0} z}{\left(\zeta_{0}-z\right)^{2}}+A(z) \frac{\zeta_{0}+z}{\zeta_{0}-z}\right\} \cdot \frac{1}{2 \pi} \int d \Psi-\frac{1}{2 \pi} \int \lambda(\zeta) d \Psi \\
& =\lambda\left(\zeta_{0}\right) \frac{1}{2 \pi} \int d \Psi-\frac{1}{2 \pi} \int \lambda(\zeta) d \Psi
\end{aligned}
$$

where the notation and justifications are as in $\S I V$. Thus, we have produced a $\lambda(\zeta)$ with the required monotonic property as shown above for $r$ satisfying (5.10). Therefore we may continue as in the proof of Theorem 5.1 and Theorem 5.9 follows.

Note. In his dissertation the author, using similar techniques, solved Problems 1 and 2 for the class $C_{d}(M)$ of convex functions in $\delta_{d}^{*}(M)$. The boundary of the extremal domain is an arc $A C$ of $\partial K_{d}$, two straight sides $A B$, $C D$, tangent to $\partial K_{d}$ at $A$ and $C$ respectively, with $B$ and $D$ either on $\partial K_{M}$ or $B=D$ (or both) and the arc $B D$ (if it exists) of $\partial K_{M}$.

Acknowledgement. The author wishes to thank Professor William Kirwan for his helpful guidance in writing this article which is a part of the author's dissertation.

\section{BIBLIOGRAPHY}

1. D. A. Brannan and W. E. Kirwan, The Maclaurin coefficients of bounded convex functions, Bull. London Math. Soc. 2 (1970), 159-164. MR 42 \#6211.

2. E. A. Coddington and N. Levinson, Theory of ordinary differential equations, McGraw-Hill, New York, 1955. MR 16, 1022.

3. G. M. Goluzin, Geometric theory of functions of a complex variable, GITTL, Moscow, 1952; English transl., Transl. Math. Monographs, vol. 26, Amer. Math. Soc., Providence, R. I., 1969. MR 15, 112; 40 \#308.

4. W. K. Hayman, Multivalent functions, Cambridge Univ. Press, New York, 1967.

5. J. A. Hummel, A variational method for starlike functions, Proc. Amer. Math. Soc. 9 (1958), 82-87. MR 20 \# 1779.

6. J. A. Jenkins, On circularly symmetric functions, Proc. Amer. Math. Soc. 6 (1955), 620-624. MR 17, 249.

7. G. Julia, Sur une équation aux dérivées fonctionelles liée à la représentation conforme, Ann. Sci. Ecole Norm. Sup. 39 (1922), 1-28. 
8. W. E. Kirwan, A note on extremal problems for certain classes of analytic functions, Proc. Amer. Math. Soc. 17 (1966), 1028-1030. MR 34 \#2854.

9. J. Krzyz, Distortion theorems for bounded convex functions. II, Ann. Univ. Mariae CurieSkłodowska Sect. A 14 (1960), 7-18. MR 25 \#4089.

10. K. Loewner, Untersuchungen über die Verserrung bei konformen Abbildungen des Einheitskreises $|z|<1$, Leipzig Berichte 69 (1917), 89-106.

11. E. Netanyahu, On univalent functions in the unit disk whose image contains a given disk, J. Analyse Math. 23 (1970), 305-322. MR 43 \#6420.

12. Ch. Pommerenke, On the Loewner differential equation, Michigan Math. J. 13 (1966), 435-443. MR 34 \#6064.

13. M. S. Robertson, Extremal problems for analytic functions with positive real part and applications, Trans. Amer. Math. Soc. 106 (1963), 236-253. MR 26 \#325.

14. T. J. Suffridge, $A$ coefficient problem for a class of univalent functions, Michigan Math. J. 16 (1969), 33-42. MR 39 \# 1646.

Department of Mathematics, University of Kentucky, Lexington, Kentucky 40506

Current address: Department of Mathematics, Texas Tech University, Lubbock, Texas 79409 